\title{
Shape Optimization and Assessment of Wind Induced
} Stresses in Domes

\author{
K. S. Babu Narayan ${ }^{1}$, Subhash C. Yaragal ${ }^{1}$ and Yukio Tamura ${ }^{2}$ \\ 1. Department of Civil Engineering, National Institute of Technology Karnataka, Mangalore 575 025, India \\ 2. Department of Architectural Engineering, Tokyo Polytechnic University, Kanagawa 243-0297, Atsugi, Japan
}

\begin{abstract}
Domes are popularly used for column free areas. They are employed as covers to reservoirs and treatment units and also as containment in nuclear reactors. Inverted domes are popular as foundations. Structures are built with an intended end use for a specified utility period. The functional requirement is to serve the purpose and the structural requirement constitutes stability, strength, safety, serviceability and durability. Satisfaction of these requirements at affordable costs is the goal of structural optimization. This paper demonstrates the need and scope for optimization of domes by formulating and solving the problem by use of calculus (volume of material is the objective function being minimized using first principles of calculus). A simple but elegant method is suggested for determination of wind stresses by statics which compares very well with that obtained by rigorous method.
\end{abstract}

Key words: Dome, shape, wind stresses, search, optimization.

\section{Introduction}

Domes, as thin shell structures, find application in a wide and varied range of structures. As cut sections of domes fit into many plan configurations they are used as roofs for column free areas. Domes are employed as covers to reservoirs and treatment units and also as containment in nuclear reactors. Inverted domes are popular as foundations.

Structures are built with an intended end use for a specified utility period. The functional requirement is to serve the purpose and the structural requirement constitutes stability, strength, safety, serviceability and durability. Satisfaction of these requirements at affordable costs is the goal of structural optimization. Realization of cost effective structures heavily relies on factors like progress made in material sciences, knowledge of behavior of materials and structural systems, analytical tools for assessment of strength, safety and serviceability, construction practices and construction economy.

Corresponding author: Subhash C. Yaragal, $\mathrm{PhD}$, associate professor, research fields: concrete technology and structural engineering. E-mail: subhashyaragal@yahoo.com.
The mode of transmission of force is a function of configuration. The technical success of optimization depends on how efficiently the load flow is accomplished. Decision making that leads to final geometry represents the highest level of structural engineering. Shells and folded plates belong to the class of stressed skin structures, which because of their geometry and small flexural rigidity of the skin, tend to carry load primarily by direct stresses acting in their plane. Shells and folded plates find application in a wide and varied range of building practice from roofing for large column free area, foundations, retaining structures to double-containment domes of nuclear structures.

Though shells of double curvature with the exception of domes are of recent origin, they are being used more in contemporary structures. Being non-developable they are more resistant to buckling and require less material. Hypars have been extensively used as the ruled surfaces can be formed easily with less expensive shuttering.

This paper presents the need and scope for optimization of domes by formulating and solving the 
problem by the very familiar method of calculus. A simples but elegant method has been suggested for the determination of wind stresses by statics and its comparison with rigorous method of analysis for low profile shells, has been made to justify its utility in arriving at a starting point for final profiling of stressed skin structures.

\section{Optimization of Domes}

An important design parameter of spherical domes is the rise to span ratio or the semi-center angle. It can be demonstrated by membrane theory that for step weight the opening angle should be less than 520 and for snow loads should be less than 450 for stresses to be compressive in Ref. [1]. The 520 angle corresponds to $\mathrm{s}$ rise to span ratio of 0.243 . For ratios less than 0.143 , the dome rather behaves as a circular slab.

High domes though membrane forces are less, consume more material owing to large surface area, whereas low domes attract more membrane forces and hence larger thickness. There is an optimum rise to span ratio for which membrane action is guaranteed and the material strength is exploited. This can be determined as outlined in the following sections.

\section{Problem Formulation and Solution}

The maximum membrane force/m by membrane theory is

$$
\frac{W R}{1+\operatorname{Cos} \phi}
$$

where $W$-self weight, $\varnothing$-opening angle, $R$-radius of dome.

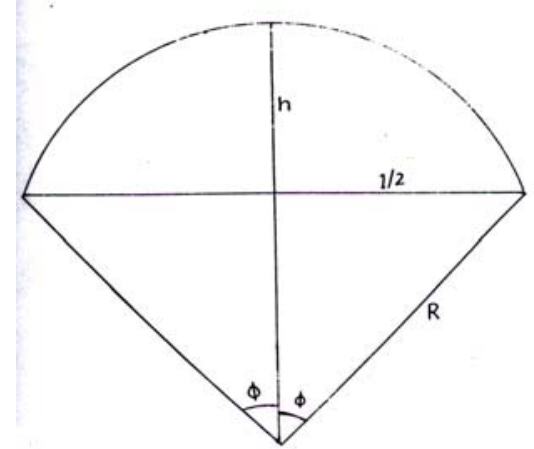

Fig. 1 Spherical dome parameters.
Assuming same thickness throughout and $f$ as permissible stress in material, the volume of material for the surface is:

$$
V=\frac{W R}{f(1+\operatorname{Cos} \phi)} \times 2 \pi R^{2}(1-\operatorname{Cos} \phi)
$$

Substituting for $R$ in terms of span as $R \operatorname{Sin} \phi=1 / 2$, we get

$$
V=\frac{2 \pi W l^{3}(1-\operatorname{Cos} \phi)}{8 f(1+\operatorname{Cos} \phi) \operatorname{Sin}^{3} \phi}
$$

Substituting $\operatorname{Sin}^{2} \phi=1-\operatorname{Cos}^{2} \phi$ the expression reduces to,

$$
\begin{gathered}
V=\frac{\pi W}{4 f} l^{3} \frac{(1-\operatorname{Cos} \phi)}{(1+\operatorname{Cos} \phi)\left(1-\operatorname{Cos}^{2} \phi\right) \operatorname{Sin} \phi} \\
V=\frac{\pi W}{4 f} \frac{l^{3}}{(1+\operatorname{Cos} \phi)^{2} \operatorname{Sin} \phi}
\end{gathered}
$$

Now volume is minimum when $(1+\operatorname{Cos} \phi)^{2} \operatorname{Sin} \phi$ is maximum, therefore

$$
\frac{d}{d \phi}(1+\operatorname{Cos} \phi)^{2} \operatorname{Sin} \phi=0
$$

On differentiating Eq. (5) we get:

$$
(1+\operatorname{Cos} \phi)^{2} \operatorname{Cos} \phi-2(1+\operatorname{Cos} \phi) \operatorname{Sin}^{2} \phi=0
$$

from which,

$$
(1+\operatorname{Cos} \phi) \operatorname{Cos} \phi-2 \operatorname{Sin}^{2} \phi=0
$$

simplifying,

$$
\begin{gathered}
\operatorname{Cos} \phi+\operatorname{Cos}^{2} \phi-2\left(1-\operatorname{Cos}^{2} \phi\right)=0 \\
3 \operatorname{Cos}^{2} \phi+\operatorname{Cos} \phi-2=0 \\
\text { or } \operatorname{Cos} \phi=2 / 3
\end{gathered}
$$

or $\phi=48.189^{\circ}$ which corresponds to a rise span ratio of 0.2236 . This satisfies all the requirements and is the optimum.

\section{Static Analysis and Wind Induced Stresses}

An approximate evaluation of maximum membrane stresses induced by wind in shells may be obtained by statics [2]. Assuming normal stress $N \phi$ to vary as $\operatorname{Cos} \theta$, where, $\theta=0$ is the wind direction [2,3], the moment of wind force about centroidal axis and the moment of $N \phi$ about the same axis are equated to determine $N \phi$.

For instance, if a low profile dome of opening angle 450 is considered it may be shown that moment of $N \phi$ about centroidal axis is

$$
\pi N_{\phi} \operatorname{Sin}^{3} \phi R^{2}
$$




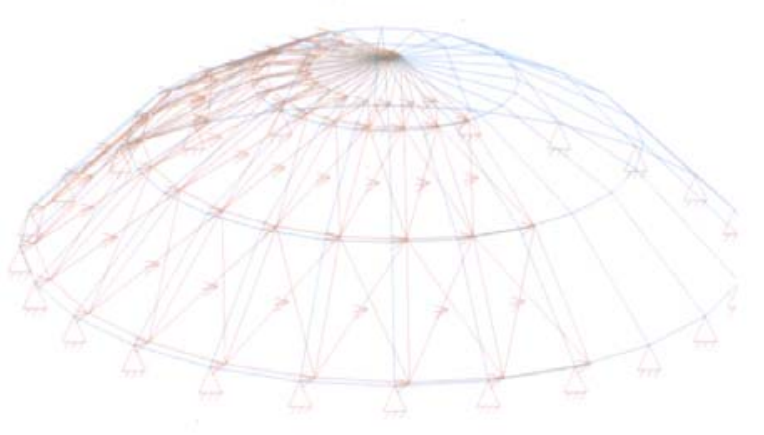

(a)

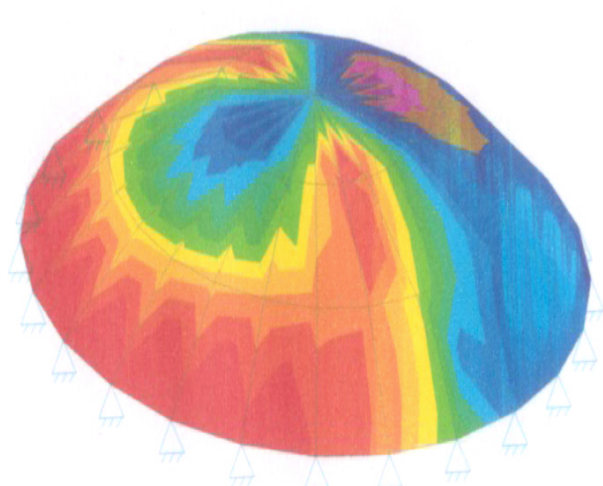

(b)

Fig. 2 Stress contours of a dome for illustration.

And the moment of wind force is

$$
\frac{1.6}{3} R^{3} p \operatorname{Sin} \phi(1-\operatorname{Cos} \phi)^{2}
$$

where, $\mathrm{p}$ is the wind pressure, equating Eqs. (11) and (12), we obtain:

$$
N_{\phi}=\frac{1.6}{3 \pi} R p\left(\frac{1-\operatorname{Cos} \phi}{\operatorname{Sin} \phi}\right)^{2}=\frac{1.6 R p}{3 \pi} \tan (\phi / 2)
$$

For $R=30 \mathrm{~m}, p=1.5 \mathrm{kN} / \mathrm{m}^{2}, \phi=450$ and $N \phi=$ $3.164 \mathrm{kN} / \mathrm{m}$ and for $0.1 \mathrm{~m}$ thickness the stress works out to be $31.64 \mathrm{kN} / \mathrm{m}^{2}$, and compares very favorably with that obtained from rigorous analysis (32.91 $\mathrm{kN} / \mathrm{m}^{2}$ ), as shown in Fig. 2. Hence utility of this method needs serious consideration at least for preliminary profiling of shells.

\section{Conclusions}

Final force distribution in membranes is dictated by the shape. Problem of finding best shape is very complex. Physical modeling and elegant soap bubble analogy have been successfully used in form finding [4]. Sophisticated computer packages are available for use by the analyst but geometry must be input. Very rough calculations are enough to get approximate forces and dimensions without placing too much weightage on mathematical rigor based on wrong or invalid assumptions. Simple approaches can gainfully be used to arrive at a good starting point for fine tuning the design problem on hand as illustrated by two examples of optimizing the span to depth ratio by classical method of calculus and determination of wind induced stresses by simple statics.

\section{References}

[1] M. Fintel, Hand Book of Concrete Engineering (2nd ed.), CBS Publishers, Delhi, 1986.

[2] M. Saluadori and M. Levy, Structural Design in Architecture (2nd ed.), Eagle Wood Cliffs, Prentice Hall, 1981.

[3] K. Mukhanov, Design of Metal Structures, Mir Publisher, Mosco, 1968.

[4] D. L. Schodek, Structures (4th ed.), Prentice Hall India, New Delhi, 2002. 